\title{
Correlation Between Glycolytic Phenotype and Tumor Grade in Soft-Tissue Sarcomas by ${ }^{18}$ F-FDG PET
}

Matthias R. Benz ${ }^{* 1}$, Sarah M. Dry ${ }^{* 2}$, Fritz C. Eilber ${ }^{3,4}$, Martin S. Allen-Auerbach ${ }^{4}$, William D. Tap ${ }^{5}$, David Elashoff ${ }^{6}$, Michael E. Phelps ${ }^{4}$, and Johannes Czernin ${ }^{4}$

${ }^{1}$ Department of Nuclear Medicine, University of Freiburg, Germany; ${ }^{2}$ Department of Pathology, David Geffen School of Medicine at the University of California Los Angeles, Los Angeles, California; ${ }^{3}$ Division of Surgical Oncology, David Geffen School of Medicine at the University of California Los Angeles, Los Angeles, California; ${ }^{4}$ Ahmanson Biological Imaging Division, Department of Molecular and Medical Pharmacology, David Geffen School of Medicine at the University of California Los Angeles, Los Angeles, California; ${ }^{5}$ Division of Medical Oncology, David Geffen School of Medicine at the University of California Los Angeles, Los Angeles, California; and ${ }^{6}$ Department of Biostatistics, David Geffen School of Medicine at the University of California Los Angeles, Los Angeles, California

Tumor glycolytic phenotyping can be accomplished with ${ }^{18} \mathrm{~F}$ FDG PET. Tumor ${ }^{18} \mathrm{~F}-\mathrm{FDG}$ uptake correlates with tumor grade in several cancers. However, the role of ${ }^{18} \mathrm{~F}-\mathrm{FDG}$ PET for the grading of soft-tissue sarcomas (STSs) warrants further research. Methods: One hundred two patients (48 men and 54 women; mean age \pm SD, $50 \pm 17$ y) with 12 STS subtypes underwent ${ }^{18} \mathrm{~F}-\mathrm{FDG}$ PET/CT before treatment. Tumor ${ }^{18} \mathrm{~F}-\mathrm{FDG}$ uptake, expressed as maximum standardized uptake value (SUVmax), was compared among subtypes and correlated with histopathologic grade. Two frequently used sarcoma grading systems - the 3-tier system of the French Federation of Cancer Centers Sarcoma Group (Fédération Nationale des Centres de Lutte Contre le Cancer [FNCLCC]) and a 2-tier system (low grade vs. high grade)—were used. Results: More than $90 \%$ of STSs (93/102) exhibited a strong glycolytic phenotype (SUV$\max , 2.7-52.2 \mathrm{~g} / \mathrm{mL}$ ). Tumor SUVmax differed significantly among tumor grades $(P<0.001$ for the 3 - and 2-tier grading systems). The FNCLCC and 2-tier grading systems predicted tumor grade with similar accuracy (area under the curve, 0.83 and 0.85 , respectively; $P=0.35$ ). SUVmax differed significantly among histologic subtypes $(P=0.03)$ in the entire population but not when high-grade STSs were analyzed separately $(P=$ 0.31). Conclusion: The tumor glycolytic phenotype correlated significantly with histologic grade as determined by both the FNCLCC and 2-tier (high vs. low) grading systems. ${ }^{18} \mathrm{~F}-\mathrm{FDG}$ PET cannot be used to reliably distinguish among grade 2 and 3 STSs (by FNCLCC) and the various subtypes.

Received Dec. 21, 2009; revision accepted Apr. 1, 2010.

For correspondence or reprints contact: Johannes Czernin, Department of Molecular and Medical Pharmacology, Ahmanson Biological Imaging Division, 10833 Le Conte Ave., Los Angeles, CA 90095-6942.

E-mail: jczernin@mednet.ucla.edu

${ }^{*}$ Contributed equally to this work.

Guest Editor: Otto C. Boerman, Radboud University Nijmegen Medical Centre

COPYRIGHT $\odot 2010$ by the Society of Nuclear Medicine, Inc.
Key Words: sarcoma; PET; ${ }^{18}$ F-FDG; glycolytic phenotype

J Nucl Med 2010; 51:1174-1181

DOI: 10.2967/jnumed.109.074229

$\mathbf{P}$ ET is increasingly used for the management of patients with soft-tissue sarcomas (STSs). Its applications include diagnosis (1) and grading with ${ }^{18} \mathrm{~F}-\mathrm{FDG}(2,3)$ or ${ }^{18} \mathrm{~F}-3$ 'deoxy-3'-fluorothymidine (4), staging (5), restaging (6), outcome predictions $(7,8)$, and treatment response monitoring $(9,10)$ with ${ }^{18} \mathrm{~F}-\mathrm{FDG}$ PET. In addition, ${ }^{18} \mathrm{~F}$-FDG PETguided biopsy (11) and the definition of target volumes for radiation therapy planning represent new opportunities for improved care of sarcoma patients.

Previous ${ }^{18} \mathrm{~F}-\mathrm{FDG}$ PET studies have reported a highly variable glycolytic phenotype of STSs that is correlated with tumor grade (3). The tumor grade has important implications for the best therapeutic strategy in sarcoma patients. Patients with low-grade sarcomas are usually treated by surgery, whereas those with high-grade variants frequently undergo combined chemo- and radiation therapy, possibly followed by surgery. Several grading systems have been used in sarcoma. A newer, frequently used method is the 3-tier system of the French Federation of Cancer Centers Sarcoma Group (Fédération Nationale des Centres de Lutte Contre le Cancer [FNCLCC]), which applies tumor type, mitotic rate, and percentage necrosis to stratify sarcomas into grade 1 (low), grade 2 (intermediate), or grade 3 (high) (12). A major strength of the French grading system is the weight placed on specific sarcoma subtypes in assigning overall grade. This approach reflects our current understanding of sarcoma biology and clinical management of this diverse group of tumors. However, the French grading system may be difficult to apply on small-core biopsies, especially for pathologists 
without specialized expertise in sarcoma diagnosis. Recently, Deyrup and Weiss proposed a 2-tier grading system (low vs. high) for biopsies, to simplify grading for nonspecialists and stratify patients into clinically relevant groups for treatment (13). The concept of using ${ }^{18}$ F-FDG PET as a noninvasive tool for tumor grading has been proposed for various cancers $(3,14,15)$. However, well-designed studies addressing the relationship between tumor glycolytic phenotype as determined by ${ }^{18} \mathrm{~F}-\mathrm{FDG}$ and the grade of STS are limited $(2,3,16)$. Thus, the role of ${ }^{18} \mathrm{~F}-\mathrm{FDG}$ PET for grading of STSs warrants further research.

Because sarcoma subtypes predict overall clinical outcome and thus heavily influence clinical management, additional diagnostic approaches for sarcomas may be useful. Specifically, if ${ }^{18}$ F-FDG PET would be able to discriminate among sarcoma subtypes, this would offer clinicians a noninvasive diagnostic tool to confirm histopathologic findings on core biopsy or fine-needle aspiration. ${ }^{18} \mathrm{~F}$-FDG PET also potentially provides an approach for more accurate tumor sampling. We and others have experienced sampling errors in sarcoma diagnosis and grading at our institution (11). Therefore, a noninvasive imaging tool that would contribute to diagnosis of sarcomas and indicate the most informative areas for biopsy would be desirable.

Thus, the aims of this study were 2-fold. First, we determined whether the degree of ${ }^{18} \mathrm{~F}$-FDG uptake correlated with tumor grade determined by the 3 -tier grading system of the FNCLCC (17) and a 2-tier grading system (low vs. high) (13). Second, we examined whether various sarcoma subtypes exhibit different glycolytic phenotypes as determined by ${ }^{18} \mathrm{~F}-\mathrm{FDG}$ PET and whether the glycolytic phenotype by ${ }^{18} \mathrm{~F}$-FDG PET can predict sarcoma grade in individual sarcoma subtypes.

\section{MATERIALS AND METHODS}

Patients with recently diagnosed STS without any therapy before PET/CT and patients with recurrent STS whose last treatment dated back at least 6 mo were eligible for this study. All patients needed to have an adequate tissue sample by coreneedle, incisional, or excisional biopsy so that tumors could be classified and graded reliably.

To avoid data contamination, patients with blood glucose levels greater than $150 \mathrm{mg} / \mathrm{dL}$ were excluded from this analysis.

\section{Clinical and Pathology Characteristics}

Between February 2005 and October 2008, 102 patients with 12 STS subtypes (Table 1) met these inclusion and exclusion criteria and were included in this study. Of these, 95 patients (93\%) were enrolled in prospective studies that were approved by the UCLA Institutional Review Board, and these patients gave written informed consent for their participation. Another 7 patients (7\%) underwent clinical PET/CT studies at UCLA. For these retrospectively enrolled patients, the consent requirements were waived by the UCLA Institutional Review Board.

\section{$\mathrm{PET} / \mathrm{CT}$}

All patients fasted for at least $6 \mathrm{~h}$ before ${ }^{18} \mathrm{~F}-\mathrm{FDG}$ PET/CT to standardize imaging conditions. Mean serum glucose levels aver- aged $97 \pm 15 \mathrm{mg} / \mathrm{dL}$ (median, $94 \mathrm{~g} / \mathrm{mL}$; range, $65-150 \mathrm{mg} / \mathrm{dL}$ ) before the injection of ${ }^{18} \mathrm{~F}-\mathrm{FDG}$. All PET/CT studies were performed using a PET/CT device consisting of a lutetium oxyorthosilicate PET scanner (ECAT ACCEL; Siemens) and a dualdetector helical CT scanner (Biograph Duo; Siemens).

CT scans were obtained with $(n=92 ; 90 \%)$ or without $(n=10$; $10 \%$ ) intravenous contrast (Omnipaque; Novaplus). Intravenous contrast was not given if medically contraindicated. Oral contrast was administered in 97 patients $(95 \%)$.

In addition to anatomic lesion localization and diagnostic information, CT images were also used for attenuation correction. The CT acquisition parameters were $130 \mathrm{kVp}, 120 \mathrm{mAs}, 1-\mathrm{s}$ tube rotation, 4-mm slice collimation, and $8-\mathrm{mm} / \mathrm{s}$ bed speed.

PET images were acquired for $1-5 \mathrm{~min} / \mathrm{bed}$ position, depending on patient body weight, $84 \pm 18 \mathrm{~min}$ after the injection of 7.77 $\mathrm{MBq}(0.21 \mathrm{mCi})$ of ${ }^{18} \mathrm{~F}-\mathrm{FDG}$ per kilogram.

Patients were instructed to use shallow breathing between the acquisition of PET and CT images, to minimize misregistration. The CT images were reconstructed using conventional filtered backprojection at 3.4-mm axial intervals, to match the slice separation of the PET data. PET images were reconstructed using iterative algorithms (ordered-subset expectation maximization; 2 iterations, 8 subsets).

\section{Image Analysis}

PET/CT images were analyzed by 1 observer who was unaware of histologic type and tumor grade. All ${ }^{18}$ F-FDG PET/CT studies were analyzed quantitatively. The single maximum pixel value (maximum standardized uptake value [SUVmax]) within the slice with the highest radioactivity concentration was detected as previously described (18). In brief, regions of interest covering the whole tumor were placed manually over every axial image plane in which tumor tissue was visualized. In this set of regions of interest, the software determined the pixel with the highest ${ }^{18} \mathrm{~F}$ FDG accumulation (SUVmax). No corrections for lean body mass were applied. In addition, the maximum tumor diameter was measured on CT images.

\section{Histopathology}

At the time of grading, all specimens were reviewed by 1 pathologist with specialty training and expertise in sarcoma pathology who was unaware of the results of the PET/CT studies. Standard diagnostic criteria for sarcoma subtyping were used (19). All STSs, except gastrointestinal stromal tumors (GISTs), were graded by the 3-tier French (FNCLCC) system (17) and by a 2-tier system (low vs. high) (13).

The FNCLCC grading system is based on 3 parameters: tumor differentiation, mitotic index, and tumor necrosis. These parameters are scored on a scale from 1 to 3 for differentiation and mitotic index and from 0 to 2 for necrosis. A 3-grade system is acquired by summing the scores obtained for each of these 3 parameters. Grade 1 is defined as a total score of 2 or 3 , grade 2 as a total of 4 or 5 , and grade 3 as a total score of 6-8 (17). For the 2-tier grading system, tumors classified as grade 2 or 3 by the French system were considered of high grade, and those classified as grade 1 were considered of low grade. This type of 2-tier grading system has been suggested as a practicable and reasonable means to providing clinicians with information to guide their therapeutic decisions (13). Additionally, it is well accepted that certain sarcoma types-for instance, angiosarcoma or malignant peripheral nerve sheath tumors-are not reliably graded by current histopathology grading 
TABLE 1. SUVmax of STS Subtypes Classified by FNCLCC Grading System

\begin{tabular}{|c|c|c|c|c|c|}
\hline Histology & No. of patients & Median & Mean & SD & Range \\
\hline \multicolumn{6}{|l|}{ Fibrous tumors } \\
\hline Desmoid grade 1 & 4 & 4.4 & 4.9 & 2.2 & $3.0-8.0$ \\
\hline Myxofibrosarcoma & 6 & 12.9 & 11.5 & 6.0 & $2.8-17.5$ \\
\hline Grade 1 & 1 & 6.2 & 6.2 & & \\
\hline Grade 2 & 5 & 15.1 & 12.5 & 6.0 & $2.8-17.5$ \\
\hline \multicolumn{6}{|l|}{ Lipomatous tumors } \\
\hline Liposarcoma & 16 & 3.6 & 8.2 & 8.9 & $1.0-31.1$ \\
\hline Myxoid & 8 & 2.8 & 4.2 & 3.7 & $1.9-13.1$ \\
\hline Grade 1 & 4 & 2.7 & 2.8 & 1.0 & $1.9-4.0$ \\
\hline Grade 2 & 4 & 3.5 & 5.5 & 5.1 & $2.1-13.1$ \\
\hline Pleomorphic grade 3 & 1 & 9.6 & 9.6 & & \\
\hline Dedifferentiated & 7 & 12.1 & 12.7 & 11.7 & $1.0-31.1$ \\
\hline Grade 2 & 4 & 2.3 & 7.1 & 10.5 & $1.0-22.9$ \\
\hline Grade 3 & 3 & 17.0 & 20.0 & 9.9 & $12.1-31.1$ \\
\hline \multicolumn{6}{|l|}{ Smooth muscle tumors } \\
\hline Leiomyosarcoma & 5 & 7.1 & 10.4 & 6.1 & $5.0-20.0$ \\
\hline Grade 2 & 2 & 6.0 & 6.0 & 1.5 & $5.0-7.1$ \\
\hline Grade 3 & 3 & 13.0 & 13.3 & 6.4 & $7.1-20.0$ \\
\hline GIST & 10 & 4.4 & 6.4 & 5.0 & $1.8-16.0$ \\
\hline Grade 1 & 4 & 3.2 & 3.4 & 1.4 & $1.9-5.2$ \\
\hline Grade 2 & 4 & 4.4 & 6.0 & 5.2 & $1.8-13.5$ \\
\hline Grade 3 & 2 & 13.0 & 13.0 & 4.1 & $10.1-16.0$ \\
\hline \multicolumn{6}{|l|}{ Skeletal muscle tumors } \\
\hline Rhabdomyosarcoma & 2 & 12.6 & 12.6 & 6.8 & $17.8-17.5$ \\
\hline Embryonal grade 3 & 1 & 7.8 & 7.8 & & \\
\hline Pleomorphic grade 3 & 1 & 17.5 & 17.5 & & \\
\hline \multicolumn{6}{|l|}{ Tumors of blood and lymph vessels } \\
\hline Angiosarcoma & 4 & 6.8 & 9.6 & 6.5 & $5.5-19.4$ \\
\hline Grade 1 & 2 & 6.0 & 6.0 & 0.7 & $5.5-6.5$ \\
\hline Grade 2 & 2 & 13.3 & 13.3 & 8.6 & $7.2-19.4$ \\
\hline \multicolumn{6}{|l|}{ Neural tumors } \\
\hline MPNST & 9 & 9.8 & 12.9 & 6.2 & $6.7-26.8$ \\
\hline Grade 2 & 5 & 9.8 & 13.5 & 8.0 & $6.7-26.8$ \\
\hline Grade 3 & 4 & 12.5 & 12.1 & 4.1 & $7.7-16.0$ \\
\hline PNET/extraskeletal Ewing sarcoma & 4 & 8.3 & 7.7 & 2.2 & $4.6-9.4$ \\
\hline Grade 2 & 1 & 9.2 & 9.2 & & \\
\hline Grade 3 & 3 & 7.4 & 7.1 & 2.4 & $4.6-9.4$ \\
\hline \multicolumn{6}{|l|}{ Miscellaneous tumors } \\
\hline Synovial sarcoma & 10 & 5.9 & 9.1 & 10.9 & $2.2-39.2$ \\
\hline Grade 2 & 7 & 7.1 & 6.4 & 2.7 & $2.9-9.3$ \\
\hline Grade 3 & 3 & 4.7 & 15.4 & 20.7 & $2.2-39.2$ \\
\hline Not otherwise specified & 31 & 10.8 & 14.9 & 11.0 & $3.2-52.2$ \\
\hline Grade 2 & 13 & 10.8 & 13.5 & 8.0 & $3.2-29.5$ \\
\hline Grade 3 & 18 & 11.6 & 16.0 & 12.9 & $3.5-52.2$ \\
\hline \multicolumn{6}{|l|}{ Extraosseous chondrosarcoma } \\
\hline Mesenchymal grade 2 & 1 & 6.2 & 6.2 & & \\
\hline
\end{tabular}

schemes $(13,20)$. In these cases, the sarcoma type and expected clinical course were given priority in assigning grade.

Four patients in our study-2 with angiosarcomas, 1 with myxofibrosarcoma, and 1 with myxoid liposarcoma-were classified as grade 1 by the French system and as high-grade in the 2-tier scheme. All 4 patients showed low numbers of mitoses (fewer than 9 per 10 high-power fields) and no tumor necrosis, thus resulting in a French grade 1 . However, because angiosarcoma grading is not reliable (20) and angiosarcomas often show aggressive behavior, these were considered of high grade in the 2-tier system.

Grading of myxofibrosarcomas is typically based on the percentage of nonmyxoid hypercellular areas (21). Because the patient with myxofibrosarcoma showed areas of hypercellularity, this patient's myxofibrosarcoma was classified as high-grade in the 2-tier system. The myxoid liposarcoma was graded on needle-core biopsy only, because an excision was not performed at UCLA. On biopsy, a small area of round-cell component was seen. This finding is significant on biopsy, because it raises the possibility that the tumor will have a significant high-grade (round-cell) component. Thus, we chose to grade this biopsy as of high-grade in the 2-tier system.

GISTs were graded according to recent consensus criteria (22). This system provides an estimated stratified risk of aggressive clinical behavior, using tumor size and mitotic activity. Several 
studies have shown that extremely low-, low-, and intermediaterisk sarcomas show significantly prolonged overall survival compared with high-risk or overtly malignant GISTs $(23,24)$. To be consistent with the analysis of the other STS subtypes, modified 2- and 3-tier grading systems were applied for GIST. For the 3-tier system, extremely low- and low-risk GISTs were classified as grade 1, intermediate-risk GISTs as grade 2, and high-risk GISTs as grade 3 tumors. For the 2-tier grading system, extremely lowand low-risk GISTs were classified as low-grade and intermediaterisk and high-risk or overtly malignant GISTs were classified as high-grade.

\section{Statistical Analysis}

Quantitative data are presented as mean $\pm \mathrm{SD}$, median, and range. The Mann-Whitney and Kruskall Wallis tests were used for unpaired comparisons between quantitative parameters. We used receiver-operating-characteristic (ROC) curves to define the optimum ${ }^{18}$ F-FDG SUVmax cutoff for predicting histopathologic grade and to measure the diagnostic accuracy of tumor ${ }^{18} \mathrm{~F}-\mathrm{FDG}$ uptake. The optimum cutoff value for the differentiation between tumor grades was defined as the point on the ROC curve with the minimum distance from the $100 \%$ true-positive and the $0 \%$ false-positive rate. Statistical analysis was performed using SPSS software (version 14.0; SPSS Inc.) for Windows (Microsoft), Statistica software (version 8.0; StatSoft, Inc.) for Windows, and GraphPad Prism software (version 5.00, GraphPad Software Inc.) for Windows. $P$ values less than 0.05 were considered statistically significant.

\section{RESULTS}

\section{Patient Characteristics}

Forty-eight men (47\%) and 54 women (53\%), with a mean age of $50 \pm 17 \mathrm{y}$ (median, $49 \mathrm{y}$; range, 18-86 y), were included in the study. Eighty-one (79\%) presented with newly diagnosed disease, and 21 patients $(21 \%)$ had recurrent disease.

\section{CT Findings}

Tumors ranged from 1 to $25 \mathrm{~cm}$ (median, $8.4 \mathrm{~cm}$; mean, $10.0 \pm 5.7 \mathrm{~cm})$. Tumor size did not differ significantly among histologic subtypes $(P=0.07)$ or tumor grade (FNCLCC, $P=0.75$; 2-tier, $P=0.52$ ).

\section{PET Findings}

The averages of tumor SUVmax for all sarcoma subtypes and grades are listed in Table 1.

The SUVmax of all tumors ranged from 1.0 to $52.2 \mathrm{~g} /$ mL. Ninety-three of 102 STSs $(91 \%)$ had an SUVmax greater than $2.5 \mathrm{~g} / \mathrm{mL}$ (median, $9.2 \mathrm{~g} / \mathrm{mL}$; mean, $11.7 \pm 8.9$ $\mathrm{g} / \mathrm{mL})$. Six of the remaining 9 lesions were liposarcomas $(2$ low-grade and 1 high-grade primary myxoid, 1 high-grade recurrent myxoid, and 2 recurrent dedifferentiated highgrade liposarcomas), 2 were GISTs (1 low-grade and 1 high-grade primary GIST), and 1 was a primary high-grade synovial sarcoma.

The SUVmax in primary malignant tumors $(n=81 ; 11.7 \pm$ $9.5 \mathrm{~g} / \mathrm{mL})$ tended to be higher than that in recurrent sarcomas $(n=21 ; 7.72 \pm 5.6 \mathrm{~g} / \mathrm{mL})(P=0.09)$.
Relationship Between ${ }^{18}$ F-FDG Uptake and

\section{Tumor Subtype}

Figure 1 shows the SUVmax of all sarcomas (represented as mean $\pm \mathrm{SD})$. SUVmax differed significantly among histologic subtypes, when tumors of all grades were included $(P=$ 0.03 ) (Fig. 1A). However, in a subgroup analysis that included only high-grade sarcomas, SUVmax did not differ significantly among the various subtypes $(P=0.31)$ (Fig. 1B).

\section{SUVmax and Tumor Grade by French System}

Ninety-two STSs were graded by the FNCLCC grading system and 10 GISTs by a modified 3-tier grading system. Fifteen (15\%) were classified as grade 1, 48 (47\%) as grade 2, and $39(38 \%)$ as grade 3 (Fig. 2A).

Tumor SUVmax differed significantly among grade 1 (mean, $4.2 \pm 1.8 \mathrm{~g} / \mathrm{mL}$; range, $1.9-8.0 \mathrm{~g} / \mathrm{mL}$ ), grade 2 (mean, $10.0 \pm 7.1 \mathrm{~g} / \mathrm{mL}$; range, $1.0-29.5 \mathrm{~g} / \mathrm{mL}$ ), and grade 3 (mean, $14.5 \pm 10.8 \mathrm{~g} / \mathrm{mL}$; range, $2.2-52.2 \mathrm{~g} / \mathrm{mL})$ tumors $(P<$ 0.001 ; Fig. 2A). By ROC curve analysis, an SUVmax threshold of $6.6 \mathrm{~g} / \mathrm{mL}$ discriminated best between grade 1 and grade 2 and 3 tumors (sensitivity and specificity of $71 \%$ and $93 \%$, respectively; area under the curve [AUC], 0.83) (Fig. 2B).

Excluding patients with recurrent disease resulted in a marginally improved AUC of 0.86 (best SUVmax threshold, $6.8 \mathrm{~g} / \mathrm{mL}$; sensitivity, 75\%; and specificity, 93\%).

However, no SUVmax threshold could be identified that reliably separated grade 1 and grade 2 tumors from grade 3 tumors (AUC, 0.70).

Within liposarcomas $(n=11$; excluding patients with recurrent disease), ${ }^{18}$ F-FDG tumor uptake differed significantly between grade 1 (mean, $2.8 \pm 1.0 \mathrm{~g} / \mathrm{mL}$ ), grade 2 (mean, $6.5 \pm 5.8 \mathrm{~g} / \mathrm{mL}$ ), and grade 3 (mean, $17.4 \pm 9.6$ $\mathrm{g} / \mathrm{mL})$ tumors $(P=0.048)$.

${ }^{18} \mathrm{~F}-\mathrm{FDG}$ uptake tended to be lower in grade 1 GISTs $(n=4)$ (mean, $3.4 \pm 1.4 \mathrm{~g} / \mathrm{mL})$ than in grade $2(n=4)$ (mean, $6.0 \pm 5.2 \mathrm{~g} / \mathrm{mL}$ ) and grade $3(n=2)$ (mean, $13.0 \pm$ $4.1 \mathrm{~g} / \mathrm{mL}$ ) GISTs. However, because of the small sample, differences in SUVmax did not reach statistical significance $(P=0.16)$.

\section{SUVmax and Tumor Grade by 2-Tier System}

Ninety-one of 102 tumors (89\%) were classified as highgrade and 11 (11\%) as low-grade (Fig. 2A). SUVmax was significantly higher in high-grade than in low-grade tumors $(11.7 \pm 9.1 \mathrm{~g} / \mathrm{mL}$ vs. $3.7 \pm 1.8 \mathrm{~g} / \mathrm{mL} ; P<0.001)$. An SUVmax of $5.2 \mathrm{~g} / \mathrm{mL}$ differentiated best between lowgrade and high-grade tumors (sensitivity, 74\%; specificity, 91; and AUC, 0.85) (Fig. 2B).

When patients with recurrent disease were excluded, the accuracy of ${ }^{18} \mathrm{~F}$-FDG PET in predicting tumor grade was only marginally improved (AUC, 0.87; optimal threshold, $5.3 \mathrm{~g} / \mathrm{mL}$; sensitivity, $76 \%$; and specificity, 90\%).

Thus, the 3- and 2-tier systems predicted tumor grade in STSs with comparable accuracy when SUVmax thresholds of $6.6 \mathrm{~g} / \mathrm{mL}$ and $5.2 \mathrm{~g} / \mathrm{mL}$, respectively, were applied (AUC, 0.83 vs. $0.85 P=0.35$ ). 


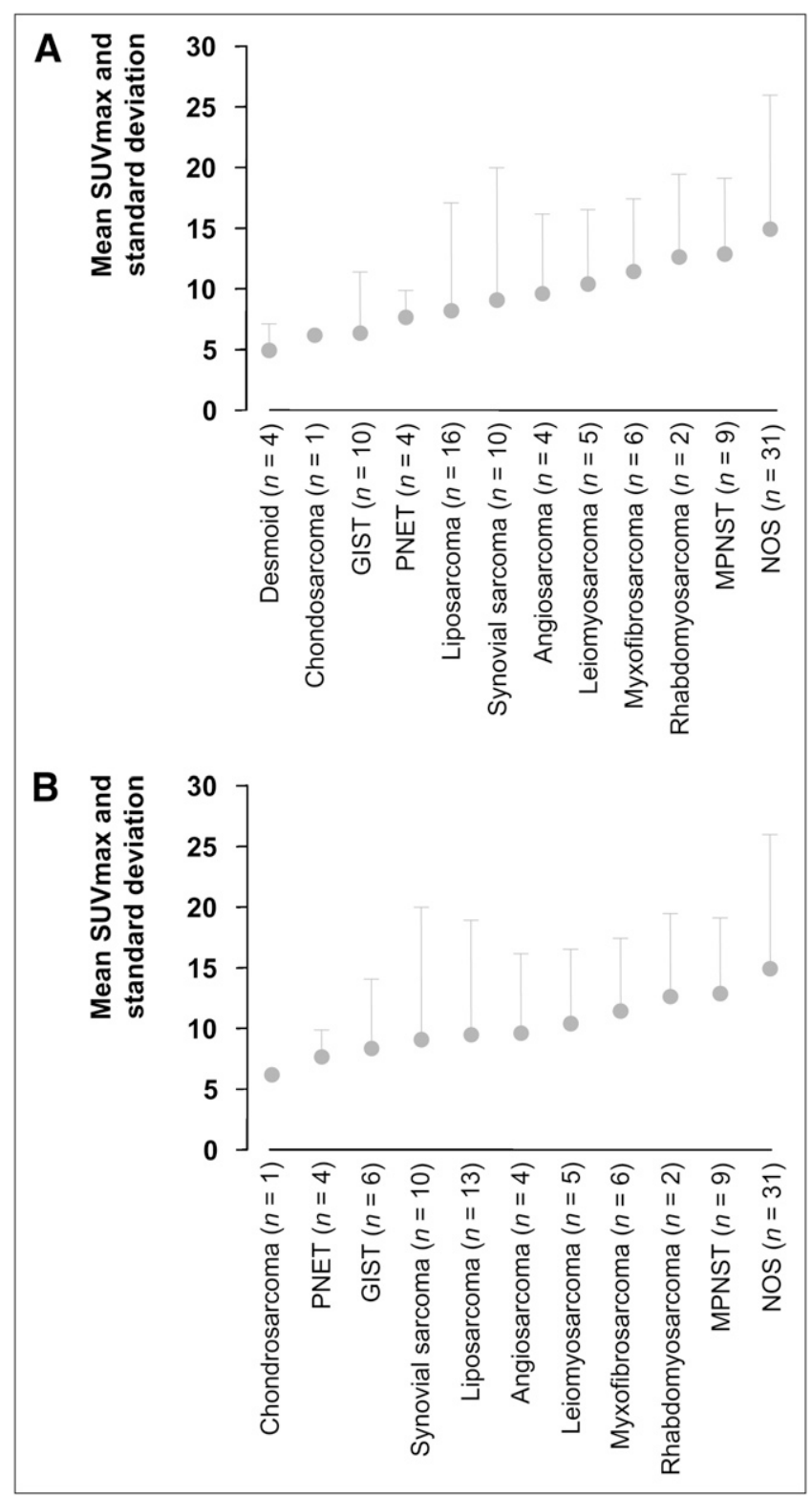

FIGURE 1. SUVmax of all malignant tumors is depicted in A as mean $\pm S D$, with 1 column for each histologic subtype. Some histologic subtypes showed higher ${ }^{18} \mathrm{~F}-\mathrm{FDG}$ uptake and wider range in SUVmax than did others $(P=0.03)$. However, in subgroup analysis (B) that included only highgrade tumors (by 2-tier grading system), SUVmax did not differ significantly among various histologic subtypes $(P=$ 0.31). MPNST = malignant peripheral nerve sheath tumors; NOS = not otherwise specified; PNET = primitive neuroectodermal tumor.

\section{DISCUSSION}

In the current study, the glycolytic phenotype correlated significantly with histologic grade as determined by 2 different grading systems - the 3-tier French (FNCLCC) grading scheme and a 2-tier grading system (high vs. low). Clinically, this correlation is relevant and significant. It is well known that histopathologic tumor grade predicts disease progression and overall survival (25-27). The correlation with tumor grade suggests that the glycolytic phenotype of STS by ${ }^{18}$ F-FDG PET may also have prognostic significance, as previously reported by Eary et al. (28). Importantly, most (>90\%) STSs in our study exhibited a strong glycolytic phenotype and were readily identified on ${ }^{18}$ F-FDG PET images. However, the degree of ${ }^{18}$ F-FDG tumor uptake could not reliably distinguish among STS subtypes. A low glycolytic phenotype (SUVmax $<2.5 \mathrm{~g} /$ $\mathrm{mL}$ ) was seen in certain STS subtypes, including myxoid liposarcomas and GISTs. Tumors with a baseline SUVmax less than $2.5 \mathrm{~g} / \mathrm{mL}$ (close to background activity) are prone to image noise and render less reliable response assessments. Because our results suggest that more than $90 \%$ of STSs exhibit sufficient ${ }^{18}$ F-FDG uptake, anticancer drug efficacy can be imaged using serial ${ }^{18}$ F-FDG PET. However, 6 of 16 patients with liposarcomas (38\%) had an SUVmax less than $2.5 \mathrm{~g} / \mathrm{mL}$. Thus, ${ }^{18} \mathrm{~F}-\mathrm{FDG}$ PET-based treatment monitoring might be difficult in some sarcoma subtypes.

Currently, most STS are initially diagnosed by CT-guided core-needle biopsy or even, in some centers, fine-needle aspiration. Although highly sensitive overall, these small biopsies may be nondiagnostic or may erroneously provide a lower tumor grade because of sampling error $(13,29,30)$. In our practice, we have, on occasion, encountered such sampling errors. In 1 previously reported case (11), core-needle biopsy of a thigh mass in a neurofibromatosis 1 patient showed neurofibroma. However, ${ }^{18}$ F-FDG PET studies showed a focal area of intense uptake. Excision showed a high-grade malignant peripheral nerve sheath tumor arising in a neurofibroma. Future studies will examine whether ${ }^{18} \mathrm{~F}-$ FDG PET in sarcoma will help to identify the highest-grade lesions within frequently large sarcoma lesions.

The relationship between sarcoma grade and ${ }^{18} \mathrm{~F}-\mathrm{FDG}$ uptake has been explored in 2 meta-analyses $(31,32)$. Both studies concluded that the degree of ${ }^{18} \mathrm{~F}$-FDG uptake, although not a perfect discriminator, was useful for differentiating low- from high-grade tumors. However, in a subgroup analysis that included only STSs, Bastiaannet et al. (31) found no significant differences between low- and high-grade STSs.

An SUVmax cutoff of 6.6 and $5.2 \mathrm{~g} / \mathrm{mL}$ predicted tumor grade in STS with the highest accuracy by the 3- and 2-tier systems, respectively. However, an SUVmax of $8.1 \mathrm{~g} / \mathrm{mL}$ correctly classified all high-grade tumors and therefore improved the specificity of this analysis (specificity, 100\%; sensitivity, 59\%).

There are several important differences between this and other studies that have correlated sarcoma grade with ${ }^{18} \mathrm{~F}-$ FDG tumor uptake. First, we showed a correlation between ${ }^{18} \mathrm{~F}-\mathrm{FDG}$ uptake and tumor grade of the entire group and within specific sarcoma subtypes (liposarcomas, GISTs). As a limitation, some of the subtype groups were too small to test for such a correlation. In contrast, prior studies reported only a correlation between grade and ${ }^{18} \mathrm{~F}-\mathrm{FDG}$ uptake within the entire group of sarcomas or a single subtype (3,16,33-35). 


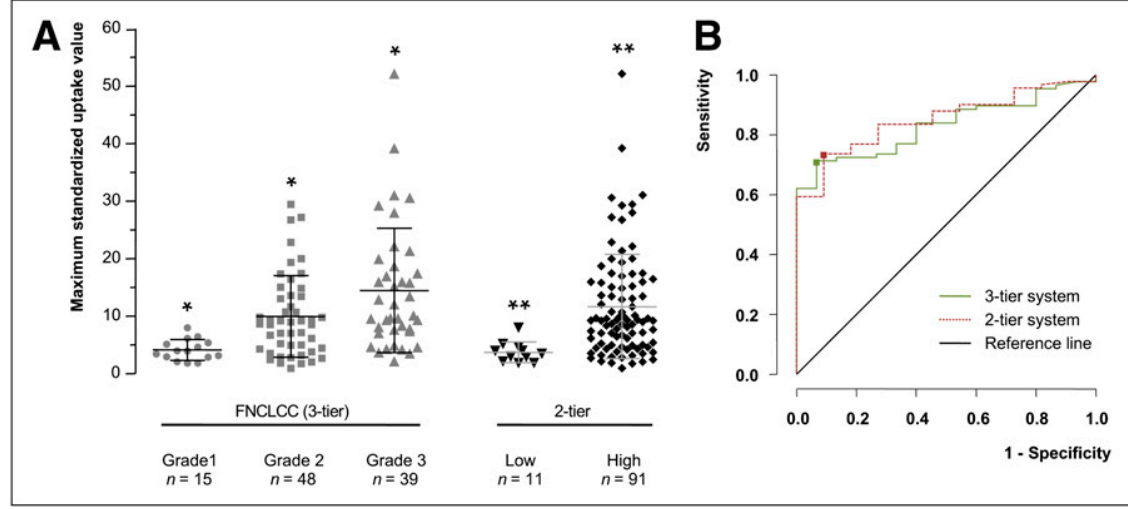

FIGURE 2. (A) Scatterplot of tumor ${ }^{18} \mathrm{~F}-\mathrm{FDG}$ uptake (SUVmax) in STSs, graded by 3 - and 2-tier systems. (B) ROC curve analysis depicts accuracy of tumor ${ }^{18} \mathrm{~F}-\mathrm{FDG}$ uptake in prediction of tumor grade assessed by 3- and 2 -tier grading systems. ${ }^{\star} P<0.001$. ${ }^{\star *} P<0.001$.
Second, this is the first study, to our knowledge, to use the French grading scheme and a proposed 2-tier scheme. Prior studies used the grading scheme of the National Cancer Institute (NCI) $(2,3)$ or did not describe the grading scheme that was used (16). We believe the French and proposed 2-tier system more accurately reflect current thinking about sarcoma biology. The French grading system greatly expanded on prior efforts by the NCI grading system to place significant weight on the specific sarcoma subtype. Several publications support the argument that the French system more accurately predicts clinical outcome than does the NCI system or other data $(36,37)$. The proposed 2-tier grading system also implicitly places significant weight on sarcoma subtype in assigning grade. For instance, the 2-tier system would not grade a synovial sarcoma as low-grade on a core biopsy, even if the tumor showed a low mitotic rate and no necrosis, because it is accepted that this subtype frequently metastasizes and requires adjuvant therapy. In any event, the current study shows that ${ }^{18} \mathrm{~F}-\mathrm{FDG}$ uptake correlates with grade as established by both systems.

Our data show a comparable glycolytic phenotype and average SUVmax among intermediate- and high-grade STSs, also supporting the argument that a simpler 2-tier grading system may be clinically useful and sufficient for determining the clinical management of patients. However, it is important to recognize that, similar to previous studies $(3,32,38,39)$, we noted considerable overlap in ${ }^{18} \mathrm{~F}$-FDG uptake between low-grade and high-grade (2-tier system) or grade 1 versus grades 2 and 3 tumors (French system). This overlap was observed within the entire sarcoma group and individual sarcoma subtypes. Therefore, whereas ${ }^{18} \mathrm{~F}-$ FDG uptake by PET provides potentially important data for initial sarcoma grading, uptake must be considered along with pathologic findings and other imaging findings.

The 12 histologic subtypes included in this study exhibited a wide range of ${ }^{18} \mathrm{~F}$-FDG tumor uptake (Fig. 1). As shown in Figure 1, sarcomas not otherwise specified and malignant peripheral nerve sheath tumors-2 subtypes with aggressive behavior and a high 5-y disease-specific mortality (40) exhibited the strongest glycolytic phenotype. Conversely, 4 myxoid liposarcomas and 2 GISTs exhibited an SUVmax less than 2.5. This result is not surprising, because myxoid liposarcomas are characterized by low tumor cellularity, likely resulting in lower total tumor glucose use (Fig. 3). SUVmax was significantly higher in dedifferentiated liposarcomas than in the primary myxoid subgroup (mean, $20.0 \pm$ $9.9 \mathrm{~g} / \mathrm{mL}$ vs. $4.4 \pm 3.9 \mathrm{~g} / \mathrm{mL}$, respectively; $P=0.03$ ). However, 2 recurrent high-grade liposarcomas also had an SUVmax less than 2.5. Thus, most but not all dedifferentiated primary liposarcomas, but not all myxoid liposarcomas, might be suitable for reliable ${ }^{18} \mathrm{~F}-\mathrm{FDG}$ PET staging or restaging and monitoring of therapeutic responses.

Desmoids are nonmetastasizing tumors and are considered low-grade sarcomas, with the potential for locally aggressive growth. Therefore, their relatively high ${ }^{18} \mathrm{~F}-\mathrm{FDG}$ uptake, with an average SUVmax of $4.9 \pm 2.2 \mathrm{~g} / \mathrm{mL}$, was

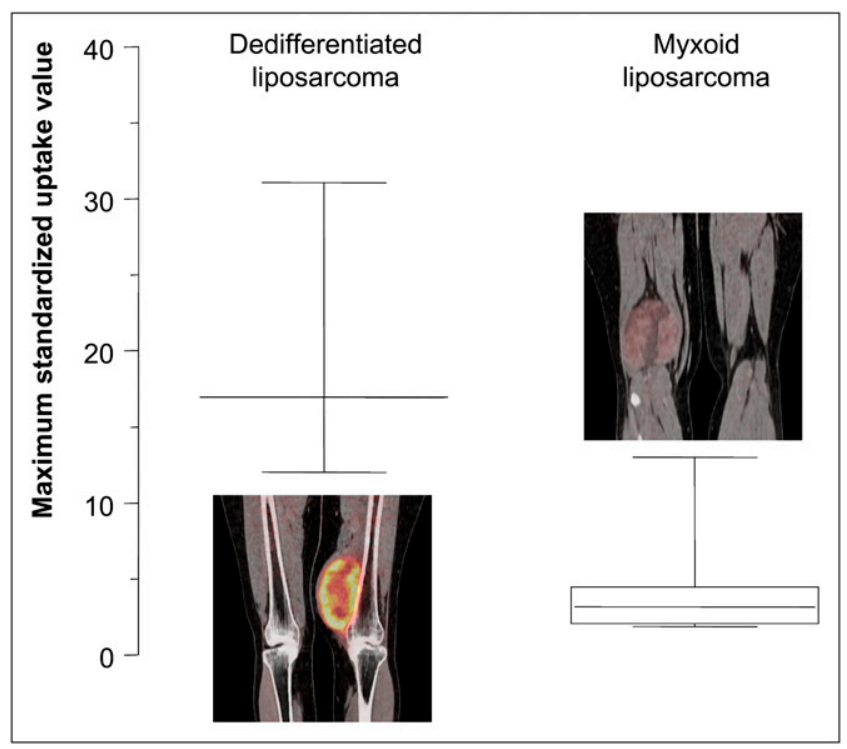

FIGURE 3. Box plots of tumor ${ }^{18} \mathrm{~F}-\mathrm{FDG}$ uptake in myxoid and dedifferentiated primary liposarcomas. Box plots depict median SUVmax and range in both subtypes. SUVmax in myxoid liposarcomas was significantly lower than SUVmax in dedifferentiated liposarcomas $(P=0.03)$. 
Primary

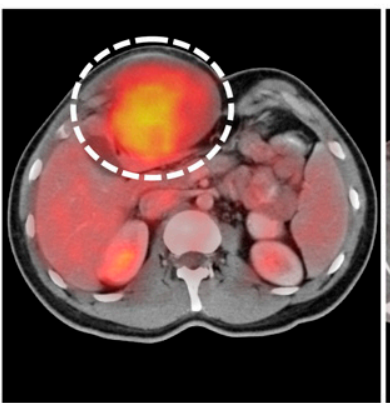

SUVmax $\quad 4.7 \mathrm{~g} / \mathrm{mL}$
Recurrent

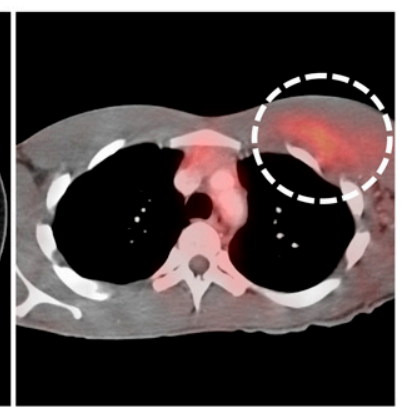

$3.0 \mathrm{~g} / \mathrm{mL}$
FIGURE 4. PET/CT images of primary and recurrent desmoid tumor.

surprising. However, desmoids are largely composed of fibroblastlike cells that are known to exhibit increased 2-deoxyglucose or ${ }^{18} \mathrm{~F}-\mathrm{FDG}$ uptake in culture. Thus, desmoids can be readily identified on ${ }^{18}$ F-FDG PET/CT (Fig. 4), despite being considered a low-grade tumor. Desmoids are readily identifiable on core biopsies; thus, higher ${ }^{18} \mathrm{~F}-\mathrm{FDG}$ uptake values should not cause clinical confusion.

These clinically relevant data will permit improved care for STS patients. First, ${ }^{18} \mathrm{~F}-\mathrm{FDG}$ PET can help guide tissue biopsy site in patients with tumors exhibiting heterogeneous ${ }^{18} \mathrm{~F}$-FDG uptake or in patients with multiple synchronous tumors. Second, our results suggest that ${ }^{18} \mathrm{~F}$-FDG PET will be helpful for monitoring low-grade STSs and in identifying the early features of dedifferentiation. Third, we show that most sarcomas exhibit a strong glucose metabolic phenotype. Therefore, ${ }^{18} \mathrm{~F}$-FDG PET allows the monitoring of treatment effects in most STS patients.

\section{CONCLUSION}

We report the first study, to our knowledge, correlating sarcoma subtype using the French 3-tier grading system (low, intermediate, and high) and a proposed 2-tier system (low and high) with ${ }^{18} \mathrm{~F}$-FDG uptake by PET. These grading systems reflect current clinical appreciation of the importance of specific sarcoma subtyping on clinical behavior. Furthermore, we believe this is the first report to detail ${ }^{18} \mathrm{~F}$ FDG uptake by grade within individual sarcoma subtypes and within the group of sarcomas as a whole. Our data indicate that for most sarcoma subtypes, ${ }^{18} \mathrm{~F}-\mathrm{FDG}$ uptake increases with grade. These observations suggest that ${ }^{18} \mathrm{~F}$ FDG PET can contribute to sarcoma grading across a variety of sarcoma subtypes. We were not able to discriminate among individual sarcoma subtypes on the basis of ${ }^{18} \mathrm{~F}$ FDG uptake alone. Thus, although ${ }^{18} \mathrm{~F}-\mathrm{FDG}$ PET offers important contributions to sarcoma grading, and possibly to the targeting of biopsy sites, diagnosis still requires tissue sampling and histopathologic analysis. Furthermore, we demonstrated a considerable overlap in ${ }^{18} \mathrm{~F}-\mathrm{FDG}$ uptake between tumors of different grades, again emphasizing the importance of histopathologic diagnosis. Importantly, we show that the proposed 2-tier grading system (low vs. high) correlates well with ${ }^{18} \mathrm{~F}-\mathrm{FDG}$ uptake, supporting the concept that this simpler grading system may be clinically useful and sufficient for determining clinical management on initial needle-core biopsies.

\section{ACKNOWLEDGMENT}

This work was supported by grants from UCLA ICMIC (5 P50 CA086306) and UCLA DOE (DE-FG0206ER64249).

\section{REFERENCES}

1. Ferner RE, Golding JF, Smith M, et al. [ $\left.{ }^{18} \mathrm{~F}\right] 2$-fluoro-2-deoxy-D-glucose positron emission tomography (FDG PET) as a diagnostic tool for neurofibromatosis 1 (NF1) associated malignant peripheral nerve sheath tumours (MPNSTs): a longterm clinical study. Ann Oncol. 2008;19:390-394.

2. Eary JF, Conrad EU, Bruckner JD, et al. Quantitative [F-18]fluorodeoxyglucose positron emission tomography in pretreatment and grading of sarcoma. Clin Cancer Res. 1998;4:1215-1220.

3. Folpe AL, Lyles RH, Sprouse JT, Conrad EU, III, Eary JF. (F-18) fluorodeoxyglucose positron emission tomography as a predictor of pathologic grade and other prognostic variables in bone and soft tissue sarcoma. Clin Cancer Res. 2000;6:1279-1287.

4. Buck AK, Herrmann K, Buschenfelde CM, et al. Imaging bone and soft tissue tumors with the proliferation marker $\left[{ }^{18} \mathrm{~F}\right]$ fluorodeoxythymidine. Clin Cancer Res. 2008;14:2970-2977.

5. Volker T, Denecke T, Steffen I, et al. Positron emission tomography for staging of pediatric sarcoma patients: results of a prospective multicenter trial. J Clin Oncol. 2007;25:5435-5441.

6. Schwarzbach MH, Dimitrakopoulou-Strauss A, Willeke F, et al. Clinical value of [18-F] fluorodeoxyglucose positron emission tomography imaging in soft tissue sarcomas. Ann Surg. 2000;231:380-386.

7. Hawkins DS, Schuetze SM, Butrynski JE, et al. $\left[{ }^{18}\right.$ F $]$ Fluorodeoxyglucose positron emission tomography predicts outcome for Ewing sarcoma family of tumors. J Clin Oncol. 2005;23:8828-8834.

8. Costelloe CM, Macapinlac HA, Madewell JE, et al. ${ }^{18} \mathrm{~F}-\mathrm{FDG}$ PET/CT as an indicator of progression-free and overall survival in osteosarcoma. $\mathrm{J} \mathrm{Nucl}$ Med. 2009;50:340-347.

9. Benz MR, Czernin J, Allen-Auerbach MS, et al. FDG-PET/CT imaging predicts histopathologic treatment responses after the initial cycle of neoadjuvant chemotherapy in high-grade soft-tissue sarcomas. Clin Cancer Res. 2009; 15:2856-2863.

10. Evilevitch V, Weber WA, Tap WD, et al. Reduction of glucose metabolic activity is more accurate than change in size at predicting histopathologic response to neoadjuvant therapy in high-grade soft-tissue sarcomas. Clin Cancer Res. 2008;14:715-720.

11. Benz MR, Czernin J, Dry SM, et al. Quantitative F18-fluorodeoxyglucose positron emission tomography accurately characterizes peripheral nerve sheath tumors as malignant or benign. Cancer. 2010;116:451-458.

12. Trojani M, Contesso G, Coindre JM, et al. Soft-tissue sarcomas of adults: study of pathological prognostic variables and definition of a histopathological grading system. Int J Cancer. 1984;33:37-42.

13. Deyrup AT, Weiss SW. Grading of soft tissue sarcomas: the challenge of providing precise information in an imprecise world. Histopathology. 2006;48:42-50.

14. Crippa F, Seregni E, Agresti R, et al. Association between $\left[{ }^{18} \mathrm{~F}\right]$ fluorodeoxyglucose uptake and postoperative histopathology, hormone receptor status, thymidine labelling index and $\mathrm{p} 53$ in primary breast cancer: a preliminary observation. Eur J Nucl Med. 1998;25:1429-1434.

15. Schoder H, Noy A, Gonen M, et al. Intensity of 18 fluorodeoxyglucose uptake in positron emission tomography distinguishes between indolent and aggressive non-Hodgkin's lymphoma. J Clin Oncol. 2005;23:4643-4651.

16. Charest M, Hickeson M, Lisbona R, Novales-Diaz JA, Derbekyan V, Turcotte RE. FDG PET/CT imaging in primary osseous and soft tissue sarcomas: a retrospective review of 212 cases. Eur J Nucl Med Mol Imaging. July 11, 2009 [Epub ahead of print]. 
17. Coindre JM. Grading of soft tissue sarcomas: review and update. Arch Pathol Lab Med. 2006;130:1448-1453.

18. Benz MR, Evilevitch V, Allen-Auerbach MS, et al. Treatment monitoring by ${ }^{18} \mathrm{~F}-$ FDG PET/CT in patients with sarcomas: interobserver variability of quantitative parameters in treatment-induced changes in histopathologically responding and nonresponding tumors. J Nucl Med. 2008;49:1038-1046.

19. Sharon W. Weiss, Goldblum JR. Enzinger and Weiss's Soft Tissue Tumors. 5th ed. New York, NY: Mosby, Elsevier, Inc.; 2008.

20. Nascimento AF, Raut CP, Fletcher CD. Primary angiosarcoma of the breast: clinicopathologic analysis of 49 cases, suggesting that grade is not prognostic. Am J Surg Pathol. 2008;32:1896-1904.

21. Mentzel T, Calonje E, Wadden C, et al. Myxofibrosarcoma: clinicopathologic analysis of 75 cases with emphasis on the low-grade variant. Am J Surg Pathol. 1996;20:391-405.

22. Demetri GD, Benjamin RS, Blanke CD, et al. NCCN Task Force report: management of patients with gastrointestinal stromal tumor (GIST) - update of the NCCN clinical practice guidelines. J Natl Compr Canc Netw. 2007;5(suppl 2):S1-S29.

23. Miettinen M, Makhlouf H, Sobin LH, Lasota J. Gastrointestinal stromal tumors of the jejunum and ileum: a clinicopathologic, immunohistochemical, and molecular genetic study of 906 cases before imatinib with long-term followup. Am J Surg Pathol. 2006;30:477-489.

24. Miettinen M, Sobin LH, Lasota J. Gastrointestinal stromal tumors of the stomach: a clinicopathologic, immunohistochemical, and molecular genetic study of 1765 cases with long-term follow-up. Am J Surg Pathol. 2005;29:52-68.

25. Coindre JM, Terrier P, Bui NB, et al. Prognostic factors in adult patients with locally controlled soft tissue sarcoma: a study of 546 patients from the French Federation of Cancer Centers Sarcoma Group. J Clin Oncol. 1996;14:869-877.

26. Gaynor JJ, Tan CC, Casper ES, et al. Refinement of clinicopathologic staging for localized soft tissue sarcoma of the extremity: a study of 423 adults. J Clin Oncol. 1992;10:1317-1329.

27. Coindre JM, Terrier P, Guillou L, et al. Predictive value of grade for metastasis development in the main histologic types of adult soft tissue sarcomas: a study of 1240 patients from the French Federation of Cancer Centers Sarcoma Group. Cancer. 2001;91:1914-1926.

28. Eary JF, O'Sullivan F, Powitan Y, et al. Sarcoma tumor FDG uptake measured by PET and patient outcome: a retrospective analysis. Eur J Nucl Med Mol Imaging. 2002;29:1149-1154.
29. Domanski HA, Akerman M, Carlen B, et al. Core-needle biopsy performed by the cytopathologist: a technique to complement fine-needle aspiration of soft tissue and bone lesions. Cancer. 2005;105:229-239.

30. Nilsson B, Bumming P, Meis-Kindblom JM, et al. Gastrointestinal stromal tumors: the incidence, prevalence, clinical course, and prognostication in the preimatinib mesylate era-a population-based study in western Sweden. Cancer. 2005;103:821-829.

31. Bastiaannet E, Groen H, Jager PL, et al. The value of FDG-PET in the detection, grading and response to therapy of soft tissue and bone sarcomas; a systematic review and meta-analysis. Cancer Treat Rev. 2004;30:83-101.

32. Ioannidis JP, Lau J. ${ }^{18} \mathrm{~F}-\mathrm{FDG}$ PET for the diagnosis and grading of soft-tissue sarcoma: a meta-analysis. J Nucl Med. 2003;44:717-724.

33. Brenner W, Conrad EU, Eary JF. FDG PET imaging for grading and prediction of outcome in chondrosarcoma patients. Eur J Nucl Med Mol Imaging. 2004; 31:189-195.

34. Brenner W, Eary JF, Hwang W, Vernon C, Conrad EU. Risk assessment in liposarcoma patients based on FDG PET imaging. Eur J Nucl Med Mol Imaging. 2006;33:1290-1295.

35. Punt SE, Eary JF, O'Sullivan J, Conrad EU. Fluorodeoxyglucose positron emission tomography in leiomyosarcoma: imaging characteristics. Nucl Med Commun. 2009;30:546-549.

36. Guillou L, Benhattar J, Bonichon F, et al. Histologic grade, but not SYT-SSX fusion type, is an important prognostic factor in patients with synovial sarcoma: a multicenter, retrospective analysis. J Clin Oncol. 2004;22:4040-4050.

37. Guillou L, Coindre JM, Bonichon F, et al. Comparative study of the National Cancer Institute and French Federation of Cancer Centers Sarcoma Group grading systems in a population of 410 adult patients with soft tissue sarcoma. J Clin Oncol. 1997;15:350-362.

38. Schulte M, Brecht-Krauss D, Heymer B, et al. Fluorodeoxyglucose positron emission tomography of soft tissue tumours: is a non-invasive determination of biological activity possible? Eur J Nucl Med. 1999;26:599-605.

39. Schulte M, Brecht-Krauss D, Heymer B, et al. Grading of tumors and tumorlike lesions of bone: evaluation by FDG PET. J Nucl Med. 2000; 41:1695-1701.

40. Eilber FC, Brennan MF, Eilber FR, Dry SM, Singer S, Kattan MW. Validation of the postoperative nomogram for 12-year sarcoma-specific mortality. Cancer. 2004;101:2270-2275. 